\title{
On Stability of the Chemotactic Dynamics in Bacterial Foraging Optimization Algorithm
}

\author{
Swagatam Das \\ Jadavpur University \\ Kolkata, India
}

\author{
Sambarta Dasgupta \\ Jadavpur University \\ Kolkata, India
}

\author{
Arijit Biswas \\ Jadavpur University \\ Kolkata, India
}

\author{
Ajith Abraham \\ Q2S, NTNU \\ Trondheim, Norway \\ ajith.abraham@ieee.org
}

\author{
Amit Konar \\ Jadavpur University \\ Kolkata, India \\ konaramit@yahoo.co.in
}

\begin{abstract}
Bacterial Foraging Optimization Algorithm (BFOA) attempts to model the individual and group behavior of E.Coli bacteria as a distributed optimization process. Since its inception, BFOA has been finding many important applications in realworld optimization problems from diverse domains of science and engineering. One key step in BFOA is the computational chemotaxis, where a bacterium (which models a candidate solution of the optimization problem) takes steps over the foraging landscape in order to reach regions with high nutrient content (corresponding to higher fitness). The simulated chemotactic movement of a bacterium may be viewed as a guided random walk or a kind of stochastic hill climbing from the viewpoint of optimization theory. In this article, we firstly derive a mathematical model for the chemotactic movements of an artificial bacterium living in continuous time. The stability and convergence-behavior of the said dynamics is then analyzed in the light of Lyapunov stability theorems. The analysis undertaken provides important insights into the search mechanism of BFOA. In addition, it indicates the necessary bounds on the chemotactic step-height parameter that avoids limit-cycles and guarantees convergence of the bacterial dynamics into an optimum. Illustrative examples as well as simulation results have been provided in order to support the analytical treatments.
\end{abstract}

\section{Categories and Subject Descriptors}

I.2.8 [Artificial Intelligence]: Problem Solving, Control Methods, and Search --- Heuristic methods; G.1.6 [Numerical Analysis]: Optimization --- Global optimization; G.3 --- Probabilistic algorithms

\footnotetext{
Permission to make digital or hard copies of all or part of this work for personal or classroom use is granted without fee provided that copies are not made or distributed for profit or commercial advantage and that copies bear this notice and the full citation on the first page. To copy otherwise, or republish, to post on servers or to redistribute to lists, requires prior specific permission and/or a fee.

CSTST 2008, October 27-31, 2008, Cergy-Pontoise, France.

Copyright 2008 ACM 978-1-60558-046-3/08/0003.\$5.00.
}

\section{General Terms \\ Algorithms}

\section{Keywords \\ Biological systems, Bacterial foraging, Computational} chemotaxis, Stability analysis, Limit cycles.

\section{INTRODUCTION}

To tackle complex search problems of real world, scientists have been looking into the nature for years, both as model and as metaphor, for inspiration. Optimization is at the heart of many natural processes like Darwinian evolution, group behavior of social insects and the foraging strategy of other microbial creatures. Natural selection tends to eliminate species with poor foraging strategies and favor the propagation of genes of species with successful foraging behavior since they are more likely to enjoy reproductive success.

Since a foraging organism or animal takes necessary action to maximize the energy utilized per unit time spent for foraging, considering all the constraints presented by its own physiology such as sensing and cognitive capabilities, and environment (e.g. density of prey, risks from predators, physical characteristics of the search space), the natural foraging strategy can lead to optimization and essentially this idea can be applied to solve real-world optimization problems. Based on this conception, Passino proposed an optimization technique, now well-known as the Bacterial Foraging Optimization Algorithm (BFOA) [1, 2]. Until date, the algorithm has successfully been applied to several real life problems like optimal controller design [1, 3], harmonic estimation [4], transmission loss reduction [5], active power filter synthesis [6], and machine learning [7]. On the algorithmic front, extensions have been made to deal with complex and multi-modal fitness landscapes, dynamical environments and to obtain efficient convergence behavior [812]. BFOA has also been hybridized with a few other state-of -the-art evolutionary computing techniques [3, 13, and 14] in order to achieve robust and efficient search performances.

One major step in BFOA is the simulated chemotactic movement. Chemotaxis is a foraging strategy that implements one type of local optimization where the bacteria try to climb 
up the nutrient concentration, avoid noxious substance and search for ways out of neutral media. This step has much resemblance with a biased random walk model [15]. The chemotactic operator employed in BFOA is supposed to guide the swarm to converge towards optima. In this article we make an attempt to find out under what conditions this local search strategy leads to a stable dynamics that can avoid limitcycles and asymptotically converge towards an optimum of the fitness landscape. The stability analysis has been undertaken using the celebrated Lyapunov's stability theorems from classical nonlinear control theory [16, 17]. Finally, we determine the bounds on the chemotactic step-size parameter $C$, which ensures asymptotic stability. Results of computer simulations have been provided in order to support the theoretical claims made in the work. Although the analysis may appear to have a limited scope, note that this article is the first of its kind and the issues of multi-bacterial population over a multi-dimensional fitness landscape are topics of further research. Here our primary objective is to gain important insight into the operational mechanism of the artificial bacterial foraging system, acting as a function optimizer.

The rest of the paper is organized as follows. Section 2 describes the classical BFOA in sufficient details. In Section 3 differential equation model governing the motion of an individual bacterium in chemotaxis phase, is derived. The model is then used to carry out stability analysis in Section 4 . Results of computer simulations have been presented and discussed in Section 5. Finally, the paper is concluded in Section 6.

\section{THE BFOA ALGORITHM}

The bacterial foraging system proceeds through four principal mechanisms, namely chemotaxis, swarming, reproduction and elimination-dispersal [1]. Below we briefly describe each of these processes and finally provide a flow chart of the complete algorithm in Figure 1.

i) Chemotaxis: This process simulates the movement of an E.coli cell through swimming and tumbling via flagella. Biologically an E.coli bacterium can move in two different ways. It can swim for a period of time in the same direction or it may tumble, and alternate between these two modes of operation for the entire lifetime. Suppose $\theta^{i}(j, k, l)$ represents $i$-th bacterium at $j$-th chemotactic, $k$-th reproductive and $l$-th elimination-dispersal step. $C(i)$ is the size of the step taken in the random direction specified by the tumble (run length unit). Then in computational chemotaxis the movement of the bacterium may be represented by

$$
\theta^{i}(j+1, k, l)=\theta^{i}(j, k, l)+C(i) \frac{\Delta(i)}{\sqrt{\Delta^{T}(i) \Delta(i)}}
$$

Where $\Delta$ indicates a unit length vector in the random direction.

ii) Swarming: An interesting group behavior has been observed for several motile species of bacteria including E.coli and $S$. typhimurium, where stable spatio-temporal patterns (swarms) are formed in semisolid nutrient medium. A group of E.coli cells arrange themselves in a traveling ring by moving up the nutrient gradient when placed amidst a semisolid matrix with a single nutrient chemo-effecter. The cells when stimulated by a high level of succinate, release an attractant aspertate, which helps them to aggregate into groups and thus move as concentric patterns of swarms with high bacterial density. The cell to cell signaling in $E$. coli swarm may be represented by the following function.

$$
\begin{aligned}
& J_{c c}(\theta, P(j, k, l))=\sum_{i=1}^{S} J_{c c}\left(\theta, \theta^{i}(j, k, l)\right) \\
& =\sum_{i=1}^{S}\left[-d_{\text {attractant }} \exp \left(-w_{\text {attractant }} \sum_{m=1}^{p}\left(\theta_{m}-\theta_{m}^{i}\right)^{2}\right)\right] \\
& +\sum_{i=1}^{S}\left[h_{\text {repellant }} \exp \left(-w_{\text {repellant }} \sum_{m=1}^{p}\left(\theta_{m}-\theta_{m}^{i}\right)^{2}\right)\right]
\end{aligned}
$$

where $J_{c c}(\theta, P(j, k, l))$ is the objective function value to be added to the actual objective function (to be minimized) to present a time varying objective function, $S$ is the total number of bacteria, $p$ is the number of variables to be optimized, which are present in each bacterium and $\theta=\left[\theta_{1}, \theta_{2, \ldots \ldots \ldots \ldots \ldots . . .} \theta_{p}\right]^{T}$ is a point in the $p$-dimensional search domain. $\quad d_{\text {aatractant }}, w_{\text {attractant }}, h_{\text {repellant }}$, and $w_{\text {repellant }}$ are different coefficients that should be chosen properly.

iii) Reproduction: The least healthy bacteria eventually die while each of the healthier bacteria (those yielding lower value of the objective function) asexually split into two bacteria, which are then placed in the same location. This keeps the swarm size constant.

iv)Elimination and Dispersal: Gradual or sudden changes in the local environment where a bacterium population lives may occur due to various reasons e.g. a significant local rise of temperature may kill a group of bacteria that are currently in a region with a high concentration of nutrient gradients. Events can take place in such a fashion that all the bacteria in a region are killed or a group is dispersed into a new location. To simulate this phenomenon in BFOA some bacteria are liquidated at random with a very small probability while the new replacements are randomly initialized over the search space.

\section{MODELING THE CHEMOTACTIC DYNAMICS}

Let us consider a single bacterium cell that undergoes chemotactic steps according to (1) over a single-dimensional objective function space. The bacterium lives in continuous time and at the $t$-th instant its position is given by $\theta(t)$. Below we list a few assumptions, which have been considered for the sake of gaining mathematical insight.

i) The objective function $J(\theta)$ is continuous and differentiable at all points in the search space. The function is uni-modal and its one and only optima (minimum) is located at $\theta=\theta_{0}$. Also $J(\theta) \neq 0$ for $\theta \neq \theta_{0}$. 
ii) The chemotactic step size $C$ is very small (Passino [1] used $\mathrm{C}=0.1$ ).

iii) The analysis applies to the regions of the fitness landscape where gradients of the function are small i.e. near to the optima.

Now, according to BFOA, the bacterium changes its position only if the modified objective function value is less than the previous one i.e. $J(\theta)>J(\theta+\Delta \theta)$ i.e. $J(\theta)-J(\theta+\Delta \theta)$ is positive. This ensures that bacterium always moves in the direction of decreasing objective function value. A particular iteration starts by generating a random vector of unit length, termed as the direction of tumble and denoted by $\Delta$. Assuming uniform rate of position change, if the bacterium moves $C \Delta$ in unit time, its position is changed by $(C \Delta)(\Delta t)$ in $\Delta t \mathrm{sec}$. We have assumed that $\Delta t$ is an infinitesimally small positive quantity, thus sign of the quantity $J(\theta)-J(\theta+\Delta \theta)$ remains unchanged if $\Delta t$ divides it. So, bacterium will change its position if and only if $\frac{J(\theta)-J(\theta+\Delta \theta)}{\Delta t}$ is positive. This crucial decision making (i.e. whether to take a step or not) activity of the bacterium can be modeled by a unit step function (also known as Heaviside step function [18]) defined as,

$$
\begin{aligned}
u(x) & =1, \text { if } x>0 \\
& =0, \text { otherwise. }
\end{aligned}
$$

Thus, $\Delta \theta=u\left(\frac{J(\theta)-J(\theta+\Delta \theta)}{\Delta t}\right) .(C \cdot \Delta)(\Delta t)$, where value of $\Delta \theta$ is 0 or $(C \Delta)(\Delta t)$ according to value of the unit step function. Dividing both sides of above relation by $\Delta t$ we get,

$$
\begin{aligned}
& \frac{\Delta \theta}{\Delta t}=u\left[\frac{J(\theta)-J(\theta+\Delta \theta)}{\Delta t}\right] C . \Delta \\
& \Rightarrow \frac{\Delta \theta}{\Delta t}=u\left[-\frac{\{J(\theta+\Delta \theta)-J(\theta)\}}{\Delta t}\right] C . \Delta
\end{aligned}
$$

Defining the velocity of the bacterium as $V_{b}=\underset{\Delta t \rightarrow 0}{\operatorname{Lim}} \frac{\Delta \theta}{\Delta t}$ (Naturally, here we assume the time to be unidirectional i.e. $\Delta t>0)$, we obtain

$$
\begin{aligned}
& V_{b}=\operatorname{Lim}_{\Delta t \rightarrow 0} \frac{\Delta \theta}{\Delta t}=\underset{\Delta t \rightarrow 0}{\operatorname{Lim}}\left[u\left\{-\frac{J(\theta+\Delta \theta)-J(\theta)}{\Delta t}\right\} . C . \Delta\right] \\
& \Rightarrow V_{b}=\operatorname{Lim}_{\Delta t \rightarrow 0}\left[u\left\{-\frac{J(\theta+\Delta \theta)-J(\theta)}{\Delta \theta} \frac{\Delta \theta}{\Delta t}\right\} . C . \Delta\right]
\end{aligned}
$$

as $\Delta t \rightarrow 0$ makes $\Delta \theta \rightarrow 0$, we may write,

$$
V_{b}=\left[u\left\{-\left(\operatorname{Lim}_{\Delta \theta \rightarrow 0} \frac{J(\theta+\Delta \theta)-J(\theta)}{\Delta \theta}\right)\left(\operatorname{Lim}_{\Delta t \rightarrow 0} \frac{\Delta \theta}{\Delta t}\right)\right\} \cdot C . \Delta\right]
$$

Again, $J(\theta)$ is assumed to be continuous and differentiable and thus $\operatorname{Lim}_{\Delta \theta \rightarrow 0} \frac{J(\theta+\Delta \theta)-J(\theta)}{\Delta \theta}$ is the value of the gradient at the point $\theta=\theta$. Therefore we have, $V_{b}=u\left(-G V_{b}\right) C \Delta$ where $G=\frac{d J(\theta)}{d \theta}=$ gradient of the objective function at $\theta=\theta$.

In (5) argument of the unit step function is $-G V_{b}$. Value of the unit step function is 1 if $G$ and $V_{b}$ are of different sign and in this case the velocity is $C \Delta$. Otherwise, it is 0 making bacterium motionless. So equation (5) suggests that bacterium will move the direction of negative gradient.

Since the unit step function $u(x)$ has a jump discontinuity at $x=0$, to simplify the analysis further, we replace $u(x)$ with the continuous logistic function $\phi(x)$, where $\phi(x)=\frac{1}{1+e^{-k x}}$.

We note that,

$$
u(x)=\operatorname{Lt}_{k \rightarrow \infty} \phi(x)=\operatorname{Lt}_{k \rightarrow \infty} \frac{1}{1+e^{-k x}}
$$

For analysis purpose $k$ cannot be infinity. We restrict ourselves to moderately large values of $k$ (say $k=10$ ) for which $\phi(x)$ fairly approximates $u(x)$. Hence from (5)

$$
V_{b}=\frac{C \Delta}{1+e^{k G V_{b}}}
$$

According to assumptions (ii) and (iii), if $C$ and $G$ are very small and $k \sim 10$, then also we may have $\left|k G V_{b}\right|<<1$. In that case we neglect higher order terms in the expansion of $e^{k g v_{b}}$ and have $e^{k g v_{b}} \approx 1+k G V_{b}$. Substituting it in (7) we obtain,

$$
\begin{aligned}
V_{b} & =\frac{C . \Delta}{2+k G V_{b}} \\
\Rightarrow V_{b} & =\frac{C \cdot \Delta}{2}\left(1-\frac{k G V_{b}}{2}\right)\left[\because\left|\frac{k G V_{b}}{2}\right|<<1\right]
\end{aligned}
$$

After some manipulation we have,

$$
V_{b}=\frac{2 C \cdot \Delta}{4+k G C \cdot \Delta}
$$

$$
\Rightarrow V_{b}=\frac{C \Delta}{2}\left(1-\frac{k G C \Delta}{4}\right)
$$

$\left[\because\left|\frac{k G C \Delta}{4}\right|=\left|\frac{k G C}{4}\right|<<1\right.$, as $\left.|\Delta|=1\right]$

$\Rightarrow V_{b}=\frac{d \theta}{d t}=-\frac{k C^{2}}{8} G+\frac{C \Delta}{2}$

$\left[\because \Delta^{2}=1\right]$

Equation (9) represents the fundamental dynamics of the computational chemotaxis step in BFOA. In what follows, our stability analysis procedures will be mostly centered on this equation.

\section{STABILITY ANALYSIS}

In this section, we analyze the stability of the chemotactic dynamics represented by (9) using the concept of Lyapunov stability theorems [16]. We begin this treatment by explaining 
some basic concepts and their interpretations from the standard literature on nonlinear control theory [17, 19].

\section{Definition 4.1}

A point $\vec{x}=\vec{x}_{e}$ is called an equilibrium state, if the dynamics of the system is given by

$$
\frac{d \vec{x}}{d t}=f(\vec{x}(t))
$$

becomes zero at $\vec{x}=\vec{x}_{e}$ for any $t$ i.e. $f\left(\vec{x}_{e}(t)\right)=0$. The equilibrium state is also called equilibrium (stable) point in $D$ dimensional hyperspace, when the state $\vec{x}_{e}$ has $D$ components.

\section{Definition 4.2}

A scalar function $V(\vec{x})$ is said to be positive definite with respect to the point $\vec{x}_{e}$ in the region $\left\|\vec{x}-\vec{x}_{e}\right\| \leq K$, if $V(\vec{x})>0$ at all points of the region except at $\vec{x}_{e}$ where it is zero.

\section{Definition 4.3}

A scalar function $V(\vec{x})$ is said to be negative definite if $-V(\vec{x})$ is positive definite.

\section{Definition 4.4}

A dynamics $\frac{d \vec{x}}{d t}=f(\vec{x}(t))$ is asymptotically stable at the equilibrium point $\vec{x}_{e}$, if

a) it is stable in the sense of Lyapunov, i.e., for any neighborhood $S(\varepsilon)$ surrounding $\vec{x}_{e} \quad(S(\varepsilon)$ contains points $\vec{x}$ for which $\left\|\vec{x}-\vec{x}_{e}\right\| \leq \varepsilon$ ) where there is a region $\mathrm{S}(\delta)(S(\delta)$ contains points $\vec{x}$ for which $\left.\left\|\vec{x}-\vec{x}_{e}\right\| \leq \delta\right), \quad \delta<\varepsilon$, such that trajectories of the dynamics starting within $S(\delta)$ do not leave $S(\varepsilon)$ as time $\mathrm{t} \rightarrow \infty$ and

b) the trajectory starting within $S(\delta)$ converges to the origin as time $t$ approaches infinity.

The sufficient condition for stability of a dynamics can be obtained from the Lyapunov's theorem, presented below.

\section{Lyapunov's stability theorem $[16,19]$}

Given a scalar function $V(\vec{x})$ and some real number $\varepsilon>0$, such that for all $\vec{x}$ in the region $\left\|\vec{x}-\vec{x}_{e}\right\| \leq \varepsilon$ the following conditions hold:

1) $V\left(\vec{x}_{e}\right)=0$

2) $\quad V(\vec{x})>0$ for $\vec{x} \neq \vec{x}_{e}$, i.e. $V(\vec{x})$ is positive definite.

3) $\quad V(\vec{x})$ has continuous first partial derivatives with respect to all components of $\vec{x}$.
Then the equilibrium state $\vec{x}_{e}$ of the system $\frac{d \vec{x}}{d t}=f(\vec{x}(t))$ is

a) asymptotically stable if $\frac{d V}{d t}<0$, i.e. $\frac{d V}{d t}$ is negative definite, and

b) asymptotically stable in the large if $\frac{d V}{d t}<0$ for $\vec{x} \neq \vec{x}_{e}, \quad$ and $\quad$ in addition, $\quad V(\vec{x}) \rightarrow \infty \quad$ as $\left\|\vec{x}-\vec{x}_{e}\right\| \rightarrow \infty$.

Remark: Lyapunov stability analysis is based on the idea that if the total energy in the system continually decreases, then the system will asymptotically reach the zero energy state associated with an equilibrium point of the system. A system is said to be asymptotically stable if all the states approach the equilibrium state with time.

\section{Theorem 4.1 (main result):}

Let the bacterial dynamics be represented by (9) and $\theta=\theta_{0}$ be the single optimum (minimum) in the region of search. Then this optimum is asymptotically stable if

$$
\begin{aligned}
& C>\frac{4}{k}\left|\frac{\theta-\theta_{0}}{J(\theta)}\right| \text { if } \theta \neq \theta_{0} \text {. } \\
& =0 \quad \text { if } \theta=\theta_{0} \text {. }
\end{aligned}
$$

\section{Proof:}

In order to determine the equilibrium point for the system, we set (by definition 3.1),

$$
\begin{gathered}
\frac{d \theta}{d t}=0 . \\
\Rightarrow-\frac{k C^{2}}{8} G+\frac{C \Delta}{2}=0 .
\end{gathered}
$$

Since the bacterium is expected to converge at the optimum of the fitness landscape, we have the equilibrium point $\theta_{e}=\theta_{0}$ and also the function gradient $G=0$ at this point. Putting $G=0$ in (13) we obtain $C=0$. Thus, the step-height $C$ should become zero at $\theta=\theta_{0}$ for the equilibrium point to be located at the desired optimum i.e.

$$
C=0 \text { if } \theta=\theta_{0}
$$

This criterion is intuitively appealing also from the perspective of an optimization algorithm. Once reaching the optimum of the uni-modal fitness landscape, the bacterium is expected to stay there and hence it should not take any more chemotactic steps or in other words it's $C$ should become zero.

Now to test the stability, consider a scalar function: $V(\theta)=\frac{k C^{2}}{8} J(\theta)-\frac{C \Delta}{2}\left(\theta-\theta_{0}\right)$,

where $J(\theta)$ is the objective function. In order to qualify as a Lyapunov energy function, $V(\theta)$ must be a positive definite function with respect to the equilibrium point $\theta_{0}$. Thus, by 
definition (4.2), $V(\theta)$ must satisfy the relation $V\left(\theta_{0}\right)=0$ and $V(\theta)>0$ if $\theta \neq \theta_{0}$.

As $C=0$ at $\theta=\theta_{0}$ we have,

$$
V\left(\theta_{0}\right)=\frac{k C^{2}}{8} J\left(\theta_{0}\right)-\frac{C \Delta}{2}\left(\theta_{0}-\theta_{0}\right)=\frac{k C^{2}}{8} J\left(\theta_{0}\right)=0
$$

Now for the second condition to be satisfied we should have,

$$
\begin{aligned}
& \frac{k C^{2}}{8} J(\theta)-\frac{C \Delta}{2}\left(\theta-\theta_{0}\right)>0 \text { for all } \theta \neq \theta_{0} . \\
\Rightarrow & \frac{k C}{4} J(\theta)>\left(\theta-\theta_{0}\right) \Delta \text { for all } \theta \neq \theta_{0} .
\end{aligned}
$$

[as $C>0$ for all positions other than optima]

Now by assumption (i), $J(\theta) \neq 0$ for all $\theta \neq \theta_{0}$, and also noting that $k>0$, dividing both sides of (16) by $\frac{k J(\theta)}{4}$ we get,

$$
C>\frac{4\left(\theta-\theta_{0}\right) \Delta}{k J(\theta)} \text { for all } \theta \neq \theta_{0} .
$$

If the right hand side of (17) be negative, it will lead to a trivial condition as step-height $C$ is always positive.

$$
\begin{aligned}
& \text { Now, }\left|\frac{4\left(\theta-\theta_{0}\right) \Delta}{k J(\theta)}\right| \geq \frac{4\left(\theta-\theta_{0}\right) \Delta}{k J(\theta)} \\
& \Rightarrow \frac{4}{k}\left|\frac{\theta-\theta_{0}}{J(\theta)}\right| \geq \frac{4\left(\theta-\theta_{0}\right) \Delta}{k J(\theta)} \quad[\text { as }|\Delta|=1]
\end{aligned}
$$

So if $C$ satisfies the relation $C>\frac{4}{k}\left|\frac{\theta-\theta_{0}}{J(\theta)}\right|$ for all $\theta \neq \theta_{0}$, then

$$
C>\frac{4}{k}\left|\frac{\theta-\theta_{0}}{J(\theta)}\right| \geq \frac{4\left(\theta-\theta_{0}\right) \Delta}{k J(\theta)}
$$

for all $\theta \neq 0$ i.e. condition (17) is automatically satisfied.

Thus, provided that $C$ satisfies conditions (14) and (17), $V(\theta)$ is a Lyapunov energy function and

$$
\frac{d V}{d t}=\frac{d V}{d \theta} \cdot \frac{d \theta}{d t} .
$$

Now, differentiating both sides of (15) with $\theta$ we have,

$$
\frac{d V}{d \theta}=\frac{k C^{2}}{8} \cdot \frac{d J(\theta)}{d \theta}-\frac{C \cdot \Delta}{2}=-\left(-\frac{k C^{2}}{8} \cdot G+\frac{C \cdot \Delta}{2}\right)
$$

Substituting values of $\frac{d V}{d \theta}$ and $\frac{d \theta}{d t}$ from (19) and (9) respectively into (18) we get,

$$
\frac{d V}{d t}=-\left(-\frac{k C^{2}}{8} G+\frac{C \Delta}{2}\right)^{2}<0, \text { if } \theta \neq \theta_{0} .
$$

Also, $\frac{d V}{d t}=0$ if $\theta=\theta_{0} \quad$ [as $C=0$ and $G=0$ at $\theta=\theta_{0}$.] Thus,

by definition (4.3), $\frac{d V}{d t}$ is negative definite. So we can infer

that the bacterial dynamics of (9) exhibits an asymptotically stable behavior with respect to the optimum $\theta=\theta_{0}$ if the step size satisfies conditions (14) and (17) simultaneously. This completes the proof.

\section{EXPERIMENTAL RESULTS}

In Section 4, we have derived the criterion for asymptotic stability of a bacterium with respect to an optimum of the search space. In this section, we investigate what happens to the dynamics of the bacterium if this criterion is met and whether the bacterium shows unstable or oscillatory behavior otherwise, with the help of computer simulations. Consider the case of a single bacterium taking chemotactic steps over one-dimensional fitness landscape of the function $J(\theta)=\theta^{2}$ where the single optimum located at $\theta=\theta_{0}=0$. Let the bacterium start from $\theta=-0.5$ and start taking chemotactic steps of height $C=0.2$ following the directives of the actual BFOA. Now as step size remains constant, condition given in (12) is violated at some point of time. Let $C_{\text {threshold }}=\frac{4}{k}\left|\frac{\theta-\theta_{0}}{J(\theta)}\right|$. Then, according to (12), the bacterium should exhibit stable dynamic behavior near the optima as long as $C>C_{\text {threshold. }}$. Table 1 shows with changing positions of bacterium varying values of $C_{\text {threshold }}$. We have assumed $k=130$.

\begin{tabular}{|c|c|c|c|}
\hline $\begin{array}{c}\text { State of phase- } \\
\text { trajectory } \\
\text { or points on } \\
\text { Figure 1 }\end{array}$ & Position & Velocity & $\begin{array}{c}\text { Direction of } \\
\text { tumble } \\
\Delta\end{array}$ \\
\hline A & -0.5 & 0.2 & +1.0 \\
\hline B & -0.3 & 0.0 & -0.1 \\
\hline C & -0.3 & 0.2 & +0.1 \\
\hline P & -0.1 & 0.2 & +0.1 \\
\hline Q & -0.1 & 0.0 & -0.1 \\
\hline S & 0.1 & -0.2 & +0.1 \\
\hline R & 0.1 & 0.0 & +0.1 \\
\hline
\end{tabular}

Table 1: Various states and set of $\Delta$

In the very first iteration, the bacterium takes a step of size 0.2 and reaches $\theta=-0.3$. Then in the second iteration it does not move (as doing so would increase the function value) and its velocity drops to 0 . This situation is represented as point $\mathrm{B}$ in phase trajectory. The line $\mathrm{AB}$ makes an angle of $-45^{\circ}$ with the position axis. Next it takes a chemotactic step. This state can be seen in C. After taking the step, it reaches P. Now the bacterium can change position by an amount $C$ or $-C$, which are 0.2 and -0.2 in this case. These cases have been shown in $\mathrm{P}$ and $\mathrm{S}$. Otherwise it remains immobile and velocity becomes 0 . These cases can be observed in $\mathrm{Q}$ and $\mathrm{R}$. The bacterium makes transition between these points in cyclic order. Here in states $\mathrm{P}, \mathrm{Q}, \mathrm{R}$, and $\mathrm{S}$, the objective function value remains constant and the distance of the bacterium from the optimum is also constant. Still it continues to change its position. From Table 1, we can predict that after reaching $\theta=-0.1$ bacterium should show asymptotically unstable behavior. Experimentally we observe that the bacterium enters stable limit cycles after reaching that position. Figure 1 
shows how the position of the bacterium $\theta$ varies with iteration time-step.

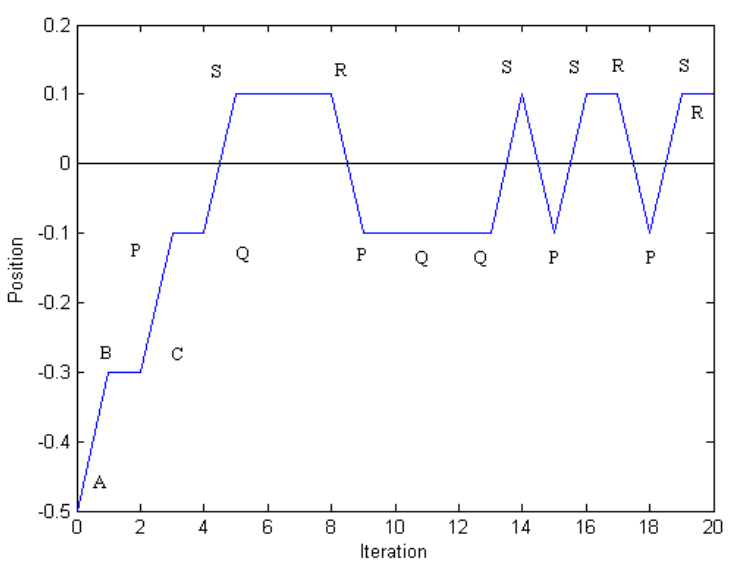

Figure 1: Variation of position with time for the bacterium.

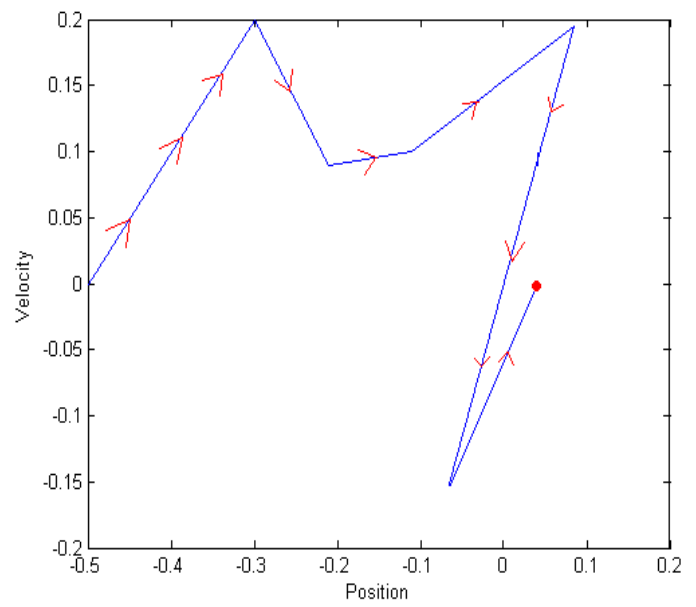

Figure 2: Phase trajectory constructed for bacterium satisfying condition (12).

Finally, we observe what happens if the condition mentioned in (12) is satisfied i.e. $C<\frac{4}{k}\left|\frac{\theta-\theta_{0}}{J(\theta)}\right|$ for all $\theta$ in the feasible search range. In this case we take $C=C_{\text {threshold }}+\xi$ for each iteration, where $\xi=0.01$ is a small positive bias. Initial position is again $\theta=-0.5$. Phase-trajectory, constructed for this case has been provided in Figure 2 and we observe it converges and shows no oscillatory behavior.

\section{CONCLUSIONS}

In this work, we have presented a simple mathematical model of the computational chemotaxis operation in BFOA, which emerges as a prominent optimization technique of current interest. The Lyapunov's stability theorems were applied to derive the conditions of asymptotic stability of a bacterium near an isolated optimum of the fitness landscape. Computer simulations over two single-dimensional uni-modal objective functions illustrate how the bacterium bursts into oscillations around the optimum instead of converging to the same, when the stability criteria derived here are not satisfied. We also note that in classical BFOA, where the step-size is usually kept constant, at some point of time the step-size violates the conditions of asymptotic stability and the bacterium starts oscillating around the optimum, instead of converging to it. This calls for some adaptation schemes, which may adjust the step-size on the run thus avoiding the limit-cycles. Future work should focus on extending the analysis undertaken here, to a multi-bacterial swarm working on a multi-dimensional fitness landscape. Another avenue is to include the effects of reproduction and elimination-dispersal events in the same mathematical model, in order to judge their effects on stability of the group dynamics. Some adaptation schemes for online adjustment of the chemotactic step-size (that guarantees convergence to the optimum) over different objective functions should also be investigated in future.

\section{REFERENCES}

[1] Passino, K. M.: Biomimicry of bacterial foraging for distributed optimization and control, IEEE Control Systems Magazine, 52-67, (2002).

[2] Liu, Y. and Passino, K. M.: Biomimicry of social foraging bacteria for distributed optimization: models, principles, and emergent behaviors, Journal of Optimization Theory And Applications: Vol. 115, No. 3, pp. 603-628, December 2002

[3] Kim, D. H., Abraham, A., Cho, J. H.: A hybrid genetic algorithm and bacterial foraging approach for global optimization, Information Sciences, Vol. 177 (18), 39183937, (2007).

[4] Mishra, S.: A hybrid least square-fuzzy bacterial foraging strategy for harmonic estimation, IEEE Trans. on Evolutionary Computation, vol. 9(1): 61-73 (2005).

[5] Tripathy, M., Mishra, S., Lai, L. L., and Zhang, Q. P.: Transmission loss reduction based on facts and bacteria foraging algorithm, Parallel Problem Solving from Nature, 222-231, (2006).

[6] Mishra, S. and Bhende C. N.: Bacterial foraging technique-based optimized active power filter for load compensation, IEEE Transactions on Power Delivery, Volume 22, Issue 1, Jan. 2007 Page(s):457 - 465.

[7] Kim, D. H. and Cho, C. H.: Bacterial foraging based neural network fuzzy learning. IICAI 2005, 2030-2036.

[8] Tang, W. J., Wu, Q. H., and Saunders, J. R.: A novel model for bacteria foraging in varying environments, ICCSA 2006, Lecture Notes in Computer Science (Vol. 3980), pp. 556-565, (2006).

[9] Li, M. S., Tang, W. J., Tang, W. H., Wu, Q. H., and Saunders, J. R.: Bacteria foraging algorithm with varying population for optimal power flow, Evo Workshops 2007, Lecture Notes in Computer Science, Vol. 4448, pp. 32-41, (2007). 
[10] Tripathy, M. and Mishra, S.: Bacteria foraging-based to optimize both real power loss and voltage stability limit, IEEE Transactions on Power Systems, Vol. 22(1), 240248, (2007).

[11] Ulagammai, L., Vankatesh, P., Kannan, P. S., and Padhy, Narayana Prasad.: Application of bacteria foraging technique trained and artificial and wavelet neural networks in load forecasting, Neurocomputing, 26592667, (2007).

[12] Munoz, Mario, A., Lopez, Jesus, A., and Caicedo, E.: Bacteria foraging optimization for dynamical resource allocation in a multizone temperature experimentation platform, Anal. and Des. of Intel. Sys. using SC Tech, ASC 41, pp.427-435, (2007).

[13] Biswas, A., Dasgupta, S., Das, S. and Abraham, A.: Synergy of PSO and Bacterial foraging optimization: a comparative study on numerical benchmarks, Second International Symposium on Hybrid Artificial Intelligent Systems (HAIS 2007), Advances in Soft computing Series, Springer Verlag, Germany, pp. 255-263, (2007).
[14] Biswas, A., Dasgupta, S., Das, S. and Abraham, A.: A synergy of differential evolution and bacterial foraging optimization for faster global search, International Journal on Neural and Mass-Parallel Computing and Information Systems - Neural Network World, Volume 17, No. 6, pp. 607-626, 2007.

[15]Hughes, B., D.: Random Walks and Random Environments, Oxford University Press, 1996.

[16] Hahn, W., Theory and Application of Liapunov's Direct Method. Prentice-Hall, Englewood Cliffs, N.J., 1963.

[17] Haddad, W.,M. and Chellaboina, V.: Nonlinear Dynamical Systems and Control: A Lyapunov-Based Approach, Princeton University Press, 2008.

[18] Anwal, R. P.: Generalized Functions: Theory and Technique, 2nd ed. Boston, MA: Birkhãuser.

[19] Kuo, B. C.: Automatic Control Systems, Prentice-Hall, Englewood Cliffs,

NJ,

1987. 